\title{
Presencia de Sarmiento en Rubén Darío
}

El viaje de Rubén Darío a Buenos Aires en calidad de cónsul general de Colombia produjo óptimos frutos. Como el poeta no tenía muchas cosas que hacer en su consulado, aprovechó el tiempo libre para familiarizarse con las letras argentinas y colaborar con más asiduidad en revistas y periódicos de aquel país.

Durante su permanencia en la capital bonaerense se publicaron dos de sus obras capitales del movimiento modernista: Los raros y Prosas profanas. Ambas constituyen dos cartas de triunfo de la nueva escuela. Además Darío colaboró en multitud de revistas: Almanaque de las Porieñas, editado por Jolly; Almanaque Peuser, ditigido por Leopo'do Díaz; Almanaque Sudamericano, de Casimiro Prieto Valdés; Argentina, de Alberto Ghiraldo; Artes y Letras, de Celestino L. Pera; Atlkintida, de Emilio Berisso; Butenos Aires, revista semanal ilustrada, de Gabriel Cantil.o; Caras y Caretas; El Búcaro de América, de Clorinda Matto de Turner; El Diario, de Manuel Lainez; El Gledicdor, de Juan Schoo; El Mercurio de América, de Eugenio Díaz Romero; El Sol y El Sol dei Domingo, de Alberto Ghiraldo; El Tiempo, de Carlos Vega Belgrano; Ilustración Argentina; Ilusisración Sudameriana; La Biblioteca, de Paul Groussac; La Nación; La Prensa; L Quincena, de Guillermo Stock; Los Principios, de Córdoba; Nosotros, de Alfredo A. Bianchi y Roberto F. Giusti; Revista Ilustrada del Río de la Plata; Revista Latina; y otras más. ${ }^{1}$

Gracias al excelente sueldo devengado por sus funciones consulares el poeta pudo gozar de una especie de bonanza económica: por vez pri. mera parecía haber alcanzado la buena "posición social" deseada desde

1 Véase Fermín Estrella Gutiérrez, "Rubén Darío a los cien años de su nacimiento", Boletin de la Academia Argentina de Letras. Buenos Aires: Tomo XXXII, Nos. 123-124 (1967), p. 101. Consúltese también a Rafael Alberto Arrieta, "Rubén Darío y la Argentina", en La Torre, Puerto Rico, nos. 55.56 (enerojunio; 1967), pp. 373-394. 
su más temprana edad. $\mathrm{Y}$ es lógico que dejara testimonio de tales circunstancias en un poema titulado "Versos de Año Nuevo":

Me pongo a pensar. . ¡ Era ayer!

Atravesaba el océano

Cónsul general colombiano.

¿Con un soñar...! Y un suponer!

¡Ah! Yo tenía oro que insufla

fuerza. Mi bolsa no era exigua.

(En asuntos de historia antigua,

Soiza Reilly es una pantufla.)

$Y$ por una coria faena

crecía la moneda vil.

En Tribuna era yo Anchorena

gracias a un Juan Cancio -al Rothschild-.2

Muchos le critican su vida bohemia pasada en la República Argentina, de la que da testimonio en otras estrofas del poema ya citado. Pero lo que hoy nos importa es la realización de una obra tan abundante como significativa por el impacto renovador que produjo en la Ciudad Cos. mópolis y en todo el ámbito de la lengua. ${ }^{3}$

Del interés que la Argentina despertó en Darío no podía estar ausente la vigorosa personalidad de Domingo Faustino Sarmiento, modelo acaso de la modernidad que nuestro poeta buscaba y símbolo admonitor del verdadero destino de la Argentina y acaso de Iberoamérica. De ahí la insistencia con que se refiere a ese constructor y maestro de pueblos.

Darío menciona a Sarmiento por vez primera en una reseña aparecida en $L a$ Nación el 22 de abril de 1894, al hablar del libro Confidencias literarias de Martín García Merou. Dice allí:

El libro concluye con dos recuerdos fúnebres: la muerte de dos gloriosos argentinos: Avellaneda y Sarmiento. Tanto las sentidas páginas sobre el primero, como la conmovedora narración de los

2 Rubén Dario, Poesías completas. Madrid: Edición Aguilar (1968), pp. 1038 1039.

3 Para un registro detallado del paso de Darío por la Argentina véase Emilio Carilla, Una etapa decisiva de Darío (Rubén Darío en la Argentina). Madrid: Gredos (1967) y Pedro Luis Barcia, Escritos dispersos de Rubén Dario (Recogidos de periódicos de Buenos Aires). La Plata: Universidad Nacional (1968). 
últimos momentos del segundo, que publicó La Nación, son en mi sentir, obras maestras.

Por todo lo cual, no puedo menos que aplaudir al autor. ${ }^{4}$

En el primer número de la Revista de América (19 de agosto de 1894), dirigida por Rubén Darío y Ricardo Jaime Freyre en Buenos Aires, hay otra referente al escritor argentino:

D. Vicente Barrantes, de la Real Academia Española, hace en La España Moderna un estudio del satírico Villergas. No es, por cierto, la delicadeza la primera de las cualidades del Sr. Barrantes. Léase lo siguiente en que se refiere a una de las más altas personalidades argentinas:

"Entre las obras restantes de nuestro satírico, nos quedan por examinar dos que estimamos de las mayores y de las menos conocidas en España. Sarmenticidio y el Juicio crítico de los poetas contemporáneos", publicadas ambas en París en 1833 y 54. Contra nuestras previsiones, en la velada que en honor de Villergas y Rodríguez Correa celebró el Ateneo el domingo 10 de este mes de junio, se dio lectura a la introducción o dedicatoria de la primera, dirigida a D. F. Sarmiento, escritor chileno, que haciendo escarnio de su apellido,

de lo más español que hay en España

y alarde. Juntamente, de la

cosa más bizarra

un Sarmiento subiéndose á la parra.

había llenado á nuestro país de denuestos, según ya se ha dicho, soeces, chabacanos y antiliterarios. ${ }^{5}$

Más tarde en un artículo publicado en $L_{a}$ Nación, el 8 de marzo de 1896, bajo el título "Menéndez y Pelayo", el poeta sale en defensa del pensador argentino, víctima de los ataques ultraconservadores del crítico español:

Han asombrado indudablemente, al sereno y elevado juicio de Me. néndez y Pelayo, las declaraciones paradojales de nuestro gran Sar-

4 E. K. Mapes, "Escritos inéditos de Rubén Dario. Nueva York: Instituto de las Españas (1938), p. 59.

5 Boyd G. Carter, La "Revista de América" de Rubén Darío y Ricardo Jaimes Freyre. Nicaragua: Imprenta Nacional, Publicaciones del centenario de Rubén Dario (1967), p. 20. Creemos que este juicio es de Rubén Darío. 
miento, cuyos gestos y maneras suelen de cuando on cuando deshacer el tocado de madama la Gramática. Pero, tenga entendido el ilustre escritor, que no es poco el bien hecho por Sarmiento a las nuevas generaciones americanas al ponerse al frente, siquiera fuese con excesiva intolerancia, de la imperante escuela netamente académica. ${ }^{6}$

La crítica a la gramática del autor de Facundo, quizás un poco descuidada por aquel argentino que preconizaba una lengua especial para su país, arreció en los ataques de Villergas (Sarmenticidio, o a mal sarmiento buena podadera) y del mismo Menéndez y Pelayo, quien opina:

En 1841 Sarmiento no era más que un periodista medio loco, que hacía continuo y fastuoso alarde de la más crasa ignorancia, y que habiendo declarado guerra a muerte al nombre español, se comp'acía en estropear nuestra lengua con toda suerte de barbarismos, afeándola con una ortografía de su propia invención. ${ }^{7}$

Darío sale por los fueros de Sarmiento, diciéndole al español, en una forma conciliadora, que las ideas de este argentino valen mucho, y que en la América polifacética hay además otros que cuidan del idioma:

Escribir las ideas de Sarmiento en la lengua de don Andrés Bello; he ahí lo que había de buscar la nueva generación. Hoy se ven los resultados de las extensas miras de Sarmiento, al sentir el influjo que el pensamiento cosmopolita ejerce sobre estas crecientes nacio. nalidades; y si existen quienes descuiden el cultivo de la lengua maternal, de nuestra hermosa lengua española, otros hay que se preocupen por ella deseando resucitar sus antiguos prestigios y aumentar razonablemente su fuerza y su gracia, en vocabulario, rítmica, plas icidad y matiz. ${ }^{8}$

En un prólogo escrito por Darío para el libro Nosotros, de Roberto J. Payró, publicado también en La Nación el 1 de mayo de 1896, alaba a este otro argentino, porque en el fondo lucha por las mismas ideas de Sarmiento:

pues tu libro es ante todo una protesta que quieres hacer contra los infecundos e impotentes, en este tiempo en que en todas par-

Mapes, Op cit., p. 91.

7 Ibid., p. 90.

8 Ibid., p. 91. 
tes, y en nuestra América sobre todo, se necesitan los fecundadores de almas, los trabajadores, los vigorosos hacedores de hijos inte. lectuales. $^{9}$

El visionario nicaragüense conocia muy bien los sinsabores sufridos por quienes se dedicaban a hacer cultura en las incipientes nacionalidades de la América hispánica de fines del siglo XIx. Por eso, en dicho pró. logo, pondera a todos los que continúan tan magna tarea de cambiar ese estado de cosas:

Tú sabes de las luchas del hombre de letras, en todos lugares atroz y martirizadora, pero en ninguna parte como en estas sociedades de la América Latina, donde el alma anda aún a tientas y la especulación del intelecto casi no tiene cabida. ${ }^{10}$

$\mathrm{Y}$ unas líneas más adelante alaba tan noble misión, tan fructífera semilla cultivada con fervor de misionero por el creador de la nueva Argentina: "Bendita sea esa voz que nos habla de trabajar y fecundar", exclama Darío, quien quería ver en Sarmiento la prefiguración de una especie de genio hispanoamericano. Por lo menos, tal se deduce del siguiente contexto de Los raros:

el Genio, en fin, que no ha tenido aún nacimiento en nuestras repúblicas, ha intentado aparecer dos veces en América: la primera en un hombre ilustre de esta tierra, la segunda en José Martí.11

Y unas páginas más adelante, en el estudio que dedica a Eugenio de Castro, en una conferencia leída en el Ateneo de Buenos Aires, el poeta dirá:

En este gran Buenos Aires que, con los ojos fijos en los Estados Unidos, al llegar a igualar a Nueva York, podrá levantar un gigantesco Sarmiento de bronce, como la Libertad de Bartholdi, la frente vuelta hacia el país de los ferrocarriles. ${ }^{12}$

9 Ibid., p. 100

10 Ibid., p. 100.

11 José Agustín Balseiro en una nota al pie de la página, nos dice "Aunque no escribe Darío el nombre de Faustino Domingo Sarmiento, la alusión al de Facundo es indispensable" en Seis estudios sobre Rubén Dario. Madrid: Gredos (1967), p. 27.

12 Rubén Dario, Los raros. Buenos Aires: Ediciones Continental (sin fecha), p. 236 . 
Darío comprende muy bien la dificultad de sintetizar la "argentinidad"; por eso comenta el libro de Payró con una gran fuerza de vidente, de alguien que ya en esa época ve la compleja heterogeneidad de la nación argentina:

Escribir un libro que contenga la condensación del ser de tu tierra. un libro al par de sociología y literatura, de estadística y de poesia, mezcla de todo y reflexión de todo; meter la Argentina en un libro, tarea es de dar temor. No hay un libro que contenga la Argentina. Yo te diré de mí, que cuando quiero confundirme con el espíritu de esta gran nación, me relaciono con el Facundo de Sarmiento, con Martín Fierro, leo los versos de Obligado, o los libros de González, y decoro [devoro] también las saludables $y$ ásperas verdades de Groussac. ${ }^{13}$

Al hablar de María Guerrero, en un artículo publicado en La Nación el 12 de junio de 1897, elogia su aparición en los escenarios del Nuevo Mundo, pero de paso apunta la separación ya existente entre España y América:

Ignacio Ramírez, en Méjico, y Sarmiento, en Buenos Aires, no sabían mucha gramática, y ya en tiempo de esos dos conductores de razas, nuestra desespañolización estaba casi realizada. España nos echó en olvido y procuró ignorarnos lo más que pudo. Naves, hombres e ideas de otros países llegaron a nuestras tierras, y nosotros nos fuimos también olvidando poco a poco de España. ${ }^{14}$

El cojo ilustrado publicó en 1899 una reseña sobre $E l$ pensamiento de América, de Luis Berisso, y allí el autor, Rubén Darío, dejó sentado lo siguiente:

Mucho habría que discurrir sobre el pensamiento de Amética, o más justamente dicho, sobre la formación de ese pensamiento. Nuestra constitución moral es tan curiosa como nuestra constitución física; el terreno es propicio y con ayuda del tiempo saldremos por completo del estado primitivo. Hablo de lo general, no de esta o aquella floración, o núcleo de formación agregadiza, verdaderas improvisaciones del tiempo. Nuestro panorama mental no deja de

13 Mapes, Op. cit., p. 101.

14 Ibid., p. 125. 
mostrar tales o cuales alturas. La vida va cambiando. Hay plantas que van desapareciendo por la influencia de las épocas. Quedan aún raros ejemplares de mégalos auros, pterodáctilos y antropopí. tecos; pero aquel cóndor se llama Andrade, aquella águila Martí, aquel hombre, Sarmiento.. ${ }^{15}$

Por el estudio dedicado a Ricardo Rojas, que aparece más tarde en su libro Cabezas, sabemos que Dario estaba enterado de que este investigador había recopilado una bibliografía sobre el autor del Facundo:

Sus calidades de pensador y de estudioso y sus disposiciones catedráticas se advierten en obras como La reskauración nacionalista, la introducción a la Bibliografía de Sarmiento, y el excelente libro sobre el abolengo de los argentinos titulado Blasón de Plata.18

En 1901 aparecen las Peregrinaciones de Dario. El poeta vive en París y tiene 34 años. Aprovecha su permanencia en la ciudad Luz para leer las diversas opiniones que publican los diarios europeos a raíz de un bus:o a Sarmiento que se estaba esculpiendo en la capital francesa (nada menos que por Rodin):

En cuanto al Sarmiento, que ha despertado en Buenos Aires las mismas tempestades que aquí el Balzac, no me es posible deciros nada. Aquí se exponen varias fotografías. Conozco las distintas opi. niones de la prensa argentina, los rudos mazazos del Sr. Groussac, los líricos y sutiles comentos de Eduardo Schiaffino y la necesidad de vigilancia policial para librar el monumento de la indignación iconoclasta. ${ }^{17}$

Este busto del expresidente argentino (es el que hoy se exhibe en los jardines de Palermo) que Francia hacía con destino a la República de Sud. américa, provocó comentarios de voces francesas como la de André Veideaux, y de ingleses como Arthur Symons. Darío recuerda entonces una bella anécdota de su permanencia en Buenos Aires, cuando conoció al nieto del gran educador y poblador de la pampa:

15 Gerald M. Moser y Hensley C. Woodbridge, "Rubén Darío y el Cojo Ilusrrado", Revista Hispánica Moderna, XXX (Enero 1964), no. 1, p. 80.

16 Rubén Dario, Cabezas. Madrid: Aguilar, Colección Crisol (1958), p. 390. Esta misma cita la encontramos en otro libro de Dario, Pensadores y artistas, pp. 67-69.

17 Rubén Darío, Peregrinaciones. Madrid: Editorial Mundo Latino, vol. XII (1918), p. 81. 
Recuerdo también algo que me refirieta en el taller de Víctor del Pol, en Buenos Aires, el nieto del ilustre luchador, Agustín Belín Sarmiento. El grande hombre alguna vez que se hablara de su estatua delante de él —ide, él estaba seguro de ella! —exclamó: "¿El mejor monumento que se me podría levantar? Ir a la Cordillera y arrancar un buen pedazo de picacho andino, y traerlo a Buenos Aires y plantarlo en donde quisieran. En la piedra bruta, en la roca viva, grabar Sarmiento: nada más." $\mathrm{Y}$ a fe que el gran original tenía razón. ${ }^{18}$

En 1902, en un prólogo que Darío escribe para un libro de Manuel Ugarte, vuelve de nuevo a invocar las doctrinas que protagonizara Sarmiento:

Nuestras repúblicas acaban de ser señaladas al mundo desde la tribuna francesa, por el ministro de instrucción pública, como futuras sostenedoras de la civilización latina. Es la idea que vibra en los versos de Andrade, en las prosas de Alberdi y de Sarmiento. ${ }^{19}$

Y en julio del mismo año, cuando publica La caravana pasa en París, tiene todavía muy presente al forjador de la moderna Argentina:

Si el Sarmiento no se parece al creador de Facundo, este Hugo sí, es idéntico al senador, á la figura conocida de todo París, que solía verse en la imperial de los ómnibus. Pero á Hugo le vió Rodin, le observó. ${ }^{20}$

En 1906, en un artículo que dedica al análisis de las revistas europeas de su tiempo, Darío elogia la Revue de Deux Mondes entre las francesas, por la calidad de sus redactores y su cosmopolitismo:

Siempre ha habido en su redacción una pluma hábil cosmopolita: antes era $M$. de Mazade, hoy es M. de Wizewa. En ella fueron juzgados, a su tiempo, los libros de Sarmiento, entre otros americanos. $^{21}$

La revista Caras y Caretas preparó un número especial dedicado a conmemorar el primer centenario de la independencia argentina, el 25

18 Ibid., p. 83. p. viii

19 Manuel Ugarte, Crónicas de Bulevar. París: Garnier Hermanos (1902),

1 Rubén Darío, Opiniones. Madrid: Editorial Mundo Latino (1918), p. 125. 
de mayo de 1910. Distinguidas personalidades como Guillermo Ferrero, Paul Adan, Max Nordeau, Víctor Marguerite, Henry de Régnier, Jules Lemaître, Paul Bourget, Máximo Gorki y o:ros, fueron entrevistados con el fin de responder a varias preguntas relacionadas con la Argenti. na. ${ }^{22}$ Darío quiso colaborar en este número, pero por razones que desconocemos, su artículo no alcanzó a llegar a la revista, y sólo más tarde, gracias a Fermín Estrella Gutiérrez, hemos conocido el contenido de este estudio titulado "La Independencia Argentina", donde hay una mención, muy significativa creemos, de Sarmien'o:

Que a la nofilia, muy natural en una nación que se forma con tanto conglomerado extranjero, se agregue una buena dosis de chauvinismo y de jingoísmo. Ayudar con puño y hombro a todo lo argentino. Con puño y hombro quiere decir, en yanqui, con mucho dinero. Ya se tiene, para comenzar, una prensa que está entre las dos o tres primeras del mundo. Es un buen comienzo. Pero que se vea más en Europa esa prensa, y los libros, y los cua. dros y las estatuas argentinas. Que no sea "yanqui" sólo por Sarmiento y por Drago: que esos señores millonarios hagan algo de lo que hacen los millonarios del Norte. ${ }^{23}$

El elogio más grande que se haya hecho en verso a los propósitos y metas de Sarmiento, lo constituye desde luego "El canto a la Argentina". Escrito por Darío en París, en 1910, es según Torres Rioseco, "la verdadera epopeya de la tierra gauchesca", ${ }^{24}$ aunque mejor sería decir que es una aprobación del progresismo civilizador de Sarmiento, El canto comienza con una inclusión del primer verso del himno nacional argentino, "Oíd mortales, oíd el grito sagrado", y se va como derramando en una generosa mención de atributos positivos en un afán de cristalizar todo lo que ese país, llamado a ser la antítesis del Coloso del Norte en la opinión de Datío, podría ofrecer a los extranjeros del mundo que viniesen a trabajar en su seno: "Todo lo ofrece la pampa con largo gesto de gravidez", agrega Torres Rioseco al citar esta estrofa:

22 Caras y Caretas, Año 13, no. 607 (25 de mayo de 1910).

23 Fermín Estrella Gutiérrez, "Una página olvidada de Rubén Darío" en Boletin de la Academia Argentina de Letras, vol. XXVII, no. 103 (1962), pp. 133-141.

24 Arturo Torres Rioseco, Rubén Darío. Cambridge: Harvard University Press, (1931), pp. 194.195. 
¡Oh Pampa! ¡Oh entraña robusta, mina del oro supremo!

He aquí que se vio la augusta resurrección de Triptolemo. En maternal continente una República ingente crea el granero del orbe, y sangre universal absorbe para dar vida al orbe entero. De ese inexhausto grancro saldrán las hostias del mañana: el hombre será, si no vana, menos multiplicada y fuerte, y será el paso de la muerte menos cruel con la especie humana.

Darío transforma en un himno profético las ideas de Sarmiento, cuya obra demuestra conocer a fondo, como lo prueban comparaciones sarmentinas que el poeta no deja de aprovechar, como aquella de la pampa con las estepas rusas:

Os espera el reino oloroso al trébol que pisa el ganado, océano de tierra sagrado al agricultor laborioso que rige el timón del arado. ¡La pampa! La estepa sin nieve, el desierto sin ser cruenta, en donde benéfico llueve riego fecundador que aumenta las demetéricas savias. Bella de honda poesía, suave de inmensidad serena, de extensa melancolía y de grave silencio plena;

La doctrina de la inmigración europea sostenida y practicada por Sarmiento, se encuentra en este poema, en donde Darío las hace suyas e invita a todos los pueblos de la tierra a habitar este inmenso granero: 
¡Dad a todas las almas abrigo, sed nación de naciones hermana; convidad a la fiesta del trigo, al domingo del lino y la lana, thanks-giving, yon Kipour, romería, la confraternidad de destinos, la confraternidad de oraciones, la confraternidad de canciones, bajo los colores argentinos! ${ }^{\mathbf{2 5}}$

Estas ideas patrióticas y progresistas que Dario expresa ahora, coinciden con pensamientos similares del poeta, manifestados anteriormente en un programa periodístico, "La Unión", donde hay sentencias que parecen escritas por Sarmiento o por Martí, como aquella en que alude a la intemporalidad de las ideas, a su eternidad:

Este y otros ejemplos nos dan a conocer que las ideas no desaparecen. Brillan, palidecen, parecen extinguirse por completo y vuelven a manifestarse con más fuerza y brillantez, porque las ideas que el patriatismo sustenta, ideas a cuyo prestigio buscan los hombres la felicidad y engrandecimiento de los pueblos, son como la ex. presión de la voluntad de Dios. ${ }^{26}$

Como representante y editor de la revista Mundial y Elegancias, de París, Darío hizo una gira por la América del Sur. El 8 de agosto de 1912 se le ofrece una gran recepción en Buenos Aires. En esa oportunidad el poeta pronuncia una conferencia sobre "Mitre y las letras", donde alude de nuevo a Sarmiento:

Son Mitre, Sarmiento, López, Gutiérrez, Echeverria, Rivera Indarte, Frías, los Varela, Mármol, Tejedor y otros tantos que han dejado páginas extraordinarias. $Y$ algunos alcanzaron la dicha de ver la patria libre y otros como Sarmiento y Mitre, vivieron para la gloria de las instituciones y del espíritu argentino, hasta completar la visión magnífica de los hombres de $1810 .{ }^{27}$

25 Rubén Dario, Poesias completas, pp. 795-825.

26 Rubén Darío, Crónica política. Madrid: Obras completas ordenadas y prologadas por Alberto Chiraldo, vol. XI (sin fecha), pp. 24-25.

27 Edelberto Torres, La dramática vida de Rubén Darío. Barcelona-México: Biografías Gandesa, 4a. edición (1966), p. 451. 
Ahora habla en forma más detallada, no sólo de su eminente patrio. tismo, sino también de sus cualidades de escritor y hasta de poeta por supuesto, en el más amplio sentido del término:

Mostraba Sarmiento su lirofobia, sin percatar que en él había prin. cipalmente un poeta, como se lo enrostrara su amigo Mitre y quizá únicamente un poeta, es decir, un creador... Y Sarmiento, dentro de su carne de estatua - pues él también tenía la famosa carne de estatua que había de cincelar Rodin - debe de haber sentido, aun contra sa voluntad, que él mismo llevaba oculta una estrella; la que ilumina el 'Fatcundo', los 'Recuerdos de Provincia' y tantas páginas que si no están en verso son obra de poeta.. ${ }^{28}$

En seguida pasa a comparar la benéfica labor emprendida por estos dos ilustres argentinos:

Lo único que faltaba a Mitre como varón de letras, porque ello no cabía en el equilibrio de sus facu'tades, aplicadas a forjar y no a imaginar, fue lo que en un sentido antiguo llamaban los griegos "fantasias", la percepción primitiva o la palabra profética de un Sarmiento. Quizá por una fortuna extraordinaria, Mitre y Sarmiento se complementaron en la labor de labrar la nacionalidad argentina, tocándole a Mitre la dura tarea de preparar la tierra y a Sarmiento la de arrojar la semilla. Sin embargo, el símil no sería así apropiado. En realidad Mitre roturaba la gleba mientras Sarmiento en cierto modo sembraba para el futuro las creaciones de su cerebro de vidente. $Y$ sin hacer un paralelo, quiero decir que sin la acción severa, metódica y práctica de Mitre, Sarmiento no hubiera podido ser sino un poeta 'clamantis in deserto', pues no había nacido para gran caudillo, o severo pastor de pueblos. ${ }^{29}$

Como vemos por esta conferencia, Darío admiraba sobremanera a Sarmiento, sin duda porque vio en él, además del vidente y forjador de pueblos prósperos y fuertes, al escritor y al poeta.

Una de las últimas referencias de Darío a Sarmiento figura en una prosa necrológica sobre el norteamericano Charles A. Dana, a quien Darío conoció por intermedio epistolar de Martí cuando el viejo maestro del periodismo dirigía el periódico neoyorquino The Sun:

28 Ibid., pp. $453-454$.

29 Ibidem., p. 456. 
Conocía también, como pocos compatriotas suyos, todo lo relativo a la América española. Era buen admirador de Sarmiento, y supongo que Bartolomé Mitre y Vedia debe guardar buenos recuer. dos de aquel noble y excelente anglo.sajón. ${ }^{\mathbf{3 0}}$

$Y$ finalmente cuando Darío se refiere al conocimiento personal de Miguel de Unamuno, nos dice:

Me presentaron una tarde, como a un ser raro - "es genial y no usa corbata", me decían-, a don Miguel de Unamuno, a quien no le agradaba, ya en aquel tiempo, que le llamaran el sabio profesor de la Universidad de Salamanca... Cultivaba su sostenido tema de antifrancesismo. $Y$ era indudablemente un notable vasco original. El señor de Unamuno no conocía entonces a Sarmiento, y hablaba con cierto desdén, basado en pocas noticias, y en su particular humor, de las letras argentinas. ${ }^{31}$

Así damos por concluido otro aspecto muy interesante del poeta nicaragüense, quien consideró siempre a la Argentina como a su segunda patria y admiró a Domingo Faustino Sarmiento como a uno de los máxi. mos forjadores de la educación y progreso de su pais, cifra entonces de lo que podía lograr la hispanidad cultivada de América. ${ }^{32}$

\section{Université de Montreal}

\section{Publio GonzÁlez-Rodas}

${ }^{30}$ Rubén Darío, Prosa dispersa. Madrid: Editorial Mundo Latino ( $\sin$ fecha), p. 83.

31 Rubén Dario, Autobiografía. Madrid: Editorial Mundo Latino (1920), p. 173.

32 Hay otros estudios sobre Darío en la Argentina: Arturo Capdevila, "Rubén Darío en Córdova de la Argentina". Revista de América. Bogotá, vol. III, no. 9 (septiembre 1945), pp. 373-376; Emilio Carilla, "Rubén Dario en Buenos Aires", Revista Interamericana de Bibliografía, XVII (1967), PP. 174-182; José A. Oría, "Rubén Darío y la Argentina", Boletín de la Academia Argentino de Letras, XXXII, nos. 123-124 (enero-junio, 1967), pp. 137-161; Torres Rioseco, "Rubén Darío en la Argentina", Nosotros, Buenos Aires, LXXIII (Nov.-Dic. 1931), pp. 325-8. 
